\title{
Projeto MagLev Cobra - Levitação Supercondutora para Transporte Urbano
}

\author{
MagLev Cobra Project: Superconducting Levitation for Public Transportation
}

\author{
Wescley T. B. de Sousa, Richard M. Stephan, Felipe S. Costa, Elkin F. Rodriguez, Flávio G. R. \\ Martins, Roberto A. H. de Oliveira, Rubens de Andrade Jr.
}

Laboratório de Aplicações de Supercondutores, Universidade Federal do Rio de Janeiro, Rio de Janeiro, RJ, Brasil.

Recebido em 31 de maio de 2016. Revisado em 4 de julho de 2016. Aceito em 22 de julho de 2016

Neste trabalho apresentam-se as principais características do Projeto MagLev Cobra desenvolvido atualmente na Universidade Federal do Rio de Janeiro. Faz-se uma retrospectiva das técnicas de levitação magnética, destacando-se as vantagens da proposta aqui apresentada, comparativamente ao sistema roda-trilho e os VLT's (Veículos Leves sobre Trilhos). Apresenta-se a história do desenvolvimento do projeto. Detalhes técnicos, como a linha de imãs permanentes, os criostatos com supercondutores, o motor linear de tração, são discutidos. Finaliza-se destacando a sustentabilidade do projeto, tendo em vista seu impacto econômico, social e ambiental.

Palavras-chave: Levitação Magnética, Supercondutores, Transporte Urbano

This work presents the main properties of the MagLev Cobra Project, currently in development at Federal University of Rio de Janeiro. One presents the basics on magnetic levitation technique, highlighting the advantages of the proposed method faced to wheel/rail systems and light rail vehicles. The history of the project is presented. Technical details, such as the line of permanent magnets, the cryostats with superconductors, the linear traction motor, are discussed. Finalizing, the sustainability of the project is highlighted, considering its economic, social and environmental impacts.

Keywords: Magnetic Levitation, Superconductivity, Urban Transport

\section{Introdução}

A necessidade de um meio de transporte público eficiente, não poluente e com moderados custos de construção é uma das prioridades do mundo moderno, onde a maior parte da população vive em grandes cidades. A procura por um meio de transporte que atendesse a estas necessidades levou a comunidade científica a desenvolver veículos baseados em levitação magnética (MagLev -Magnetic Levitation) e não mais nos convencionais modelos roda-trilho, que limitam as capacidades operacionais dos veículos [1].

Existem três principais técnicas de levitação para veículos de transporte MagLev: levitação eletrodinâmica, levitação eletromagnética e levitação supercondutora. Todas estas técnicas tem sido empre-

*Endereço de correspondência: wescley@daad-alumni.de gadas para a levitação de trens de alta velocidade ou urbanos. Poucos projetos, porém, empregam levitação supercondutora. Neste trabalho, apresentaremos uma breve introdução às levitações eletrodinâmica e eletromagnética e daremos enfoque maior na levitação supercondutora.

O projeto Maglev Cobra, atualmente desenvolvido pelo Laboratório de Aplicações de Supercondutores (LASUP) na Universidade Federal do Rio de Janeiro (UFRJ) é baseado na levitação supercondutora e propõe um veículo levitado por forças magnéticas composto de curtos módulos, permitindo curvas com até $30 \mathrm{~m}$ de raio, rampas de $15 \%$ de elevação e velocidades de até $70 \mathrm{~km} / \mathrm{h}$. A movimentação do veículo deverá se assemelhar ao serpentear de uma cobra, sugerindo o nome MagLev Cobra.

As forças de levitação surgem do diamagnetismo e aprisionamento de fluxo magnético característicos 
de materiais supercondutores de alta temperatura crítica. Blocos de óxido de ítrio-bário-cobre (YBCO) são utilizados sobre imãs de Nd-Fe-B.

Supercondutores de alta temperatura crítica foram descobertos no fim do século XX [2] e até hoje não há um uso industrial ou em larga escala de tais materiais. No entanto, perspectivas de inovação, originalidade e desenvolvimentos tecnológicos frequentemente são anunciados.

Uma das vantagens da levitação supercondutora reside na sua independência de sistemas roda-trilho convencionais, anulando o atrito com o solo, reduzindo o desgaste e o custo de manutenção do veículo. Para a tração do MagLev, um motor elétrico de indução linear é usado. Aliado a tecnologias de frenagem regenerativa, que devolve para a rede elétrica grande parte da energia utilizada para a aceleração, e painéis fotovoltaicos nas estações o MagLev apresenta um rendimento alto sem emissão de poluentes.

O nível de ruído emitido por este veículo é baixo, o que lhe permite trafegar dentro das cidades em estruturas elevadas. Além disso, o custo estimado de construção é apenas $1 / 3$ daquele previsto para construção de metrôs subterrâneos.

\section{Tecnologias MagLev}

Os veículos MagLev são comumente associados a veículos de alta velocidade para transporte interurbano de longa distância. O MagLev Cobra foge desta definição comum por se destinar a transporte em perímetro urbano de baixa velocidade. Como já mencionado, existem três diferentes técnicas de levitação para trens MagLev em função da forma como as forças magnéticas atuam no processo de levitação. As primeiras ideias para esta aplicação de campos magnéticos datam da primeira metade do século $\mathrm{XX}$, mas a viabilidade destes projetos começou a ser alcançada a partir dos avanços tecnólogicos em eletrônica e sistemas digitais, na década de 1970 [3.

Após mais de 40 anos de pesquisa e desenvolvimento, as técnicas de levitação magnética podem ser sumarizadas em três grupos.

\subsection{Levitação Eletrodinâmica: Forças de Repulsão}

A levitação eletrodinâmica se baseia no mesmo princípio de funcionamento de um motor elétrico de indução. Quando um campo magnético apresenta movimento relativo a um elemento condutor, correntes elétricas são induzidas proporcionalmente a sua derivada temporal, de acordo com a Lei de Faraday-Lenz. Estas correntes induzidas, por sua vez, geram seu próprio campo magnético que se opõe ao primeiro através de uma força restaurativa, isto é, que se opõe àquela variação temporal do campo magnético. Em um MagLev eletrodinâmico esse efeito manifesta-se como uma força de repulsão vertical entre o veículo e o trilho, causando a levitação e como uma força de arraste que se opõe ao movimento. A força de repulsão é usada para a levitação e aumenta com a velocidade do veículo enquanto que a força de arraste diminui de intensidade à medida que a velocidade aumenta (figura 1).

É importante mencionar que uma força grande o bastante para levitar um veículo do porte de um trem só é alcançada a partir de determinadas velocidades. Portanto, ainda é necessário um sistema rodatrilho para os períodos de aceleração e frenagem. O Japão se destaca na aplicação desta tecnologia com uma linha experimental de quase $43 \mathrm{~km}$ em Yamanashi, que em abril de 2015 atingiu o recorde mundial de velocidade: $603 \mathrm{~km} / \mathrm{h}$ [4].

\subsection{Levitação Eletromagnética: Forças de Atração}

A levitação eletromagnética utiliza a força de atração entre um eletroímã e um material ferromagnético para a levitação. Para isso, o veículo é mantido em um equilíbrio instável, sendo atraído ora para cima (com o eletroímã ligado), ora para baixo (por ação da gravidade), oscilando milímetros em torno da altura de levitação. O controle exigido por este tipo de sistema deve ser devidamente sintonizado.

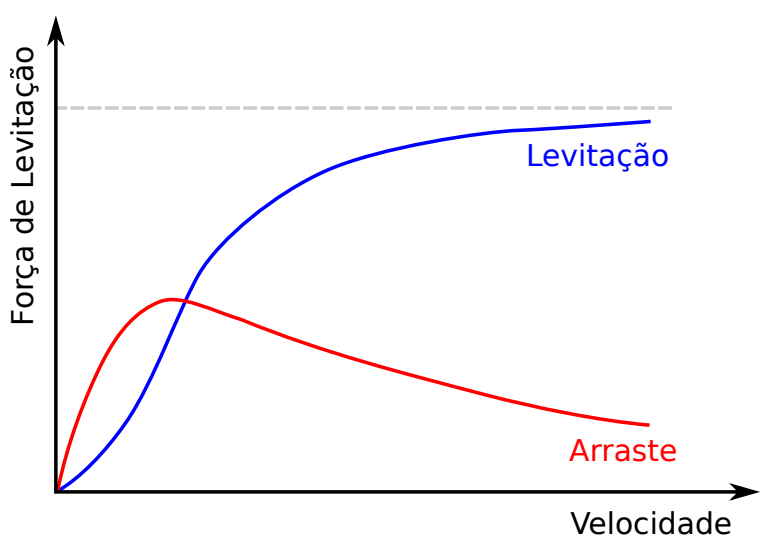

Figura 1: Forças da Levitação Eletrodinâmica. 
Esta abordagem é utilizada no Transrapid, proposta alemã de MagLev, em uma linha de $30 \mathrm{~km}$ operando desde 2003 em Xangai [5].

\subsection{Levitação Supercondutora: Forças de Atração e Repulsão}

O terceiro e último grupo é também o mais recente. Materiais supercondutores de alta temperatura crítica possuem a propriedade de aprisionar e repelir o campo magnético de forma a se manter em equilíbrio estável na presença de um campo externo constante. Isto se deve ao aprisionamento de fluxo magnético em vórtices do material supercondutor do tipo II, como mostrado na figura 2 a.

Esse efeito é aproveitado ao substituir a suspensão do veículo por mancais supercondutores e os trilhos por linhas de ímãs permanentes. Sendo a configuração de campo magnético constante ao longo da direção dos trilhos, o único grau de liberdade que o veículo tem para movimentação sem impedimento é ao longo destes. Nos demais graus, os supercondutores reagem à variação de campo magnético e automaticamente impelem forças restauradoras para a posição de origem, mantendo-se constantemente em equilíbrio estável sem qualquer contato mecânico entre as partes. A grande vantagem desse sistema é que estas forças ocorrem naturalmente, independente da ação de sensores e atuadores, como no caso eletromagnético ou da pré-existência de movimento relativo, como no caso eletrodinâmico. Estas propriedades são eficazes para transporte urbano de baixa velocidade e fácil operação.

O Brasil se destacou no cenário mundial sendo o primeiro e único, até o momento, a aplicar esta tecnologia em um veículo operacional em teste numa linha de $200 \mathrm{~m}$ no campus principal da Universidade Federal do Rio de Janeiro (UFRJ) (figura 2b).

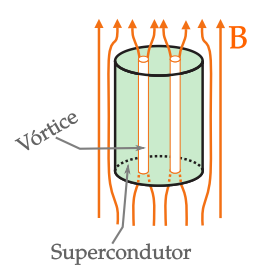

(A)

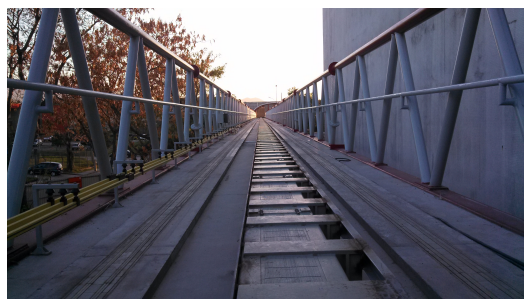

(B)
Figura 2: (A) Aprisionamento de fluxo magnético em supercondutores do tipo II e (B) linha de teste de $200 \mathrm{~m}$ construída para testes do veículo Maglev Cobra.

\subsubsection{A Força Banana}

Forças de repulsão entre ímãs permanentes e supercondutores podem não ser triviais como aparentam. Estas forças se tornam mais fortes à medida que ímã e supercondutor se aproximam. No entanto, devido ao aprisionamento de vórtices (pinning), a força de levitação em um ímã sobre um supercondutor do tipo II é diferente quando o ímã se aproxima do supercondutor do que quando o mesmo se afasta do supercondutor [6]. Quanto mais próximo do ímã o supercondutor estiver, maior será a quantidade de vórtices dentro deste. Quando se afasta o ímã do supercondutor, as forças repulsivas diminuem. Deste modo as linhas de campo aprisionadas pelo supercondutor (devido ao pinning), geram uma força de atração.

O resultado deste processo é a curva força $\mathrm{x}$ distância mostrada na figura 3 no ponto $\mathrm{Pa}$ e $\mathrm{Pb}$ as forças magnéticas compensam o peso do ímã. Se o ímã for levado para um ponto mais próximo do supercondutor e solto no ponto Pc, ele irá retornar naturalmente ao ponto de equilíbrio Pa. Do mesmo modo, se o ímã repousa sobre o supercondutor e este é resfriado abaixo de $\mathrm{T}_{c}$ (field cooling), o ímã certamente levitará na posição do ponto Pa.

Embora qualquer supercondutor possa ser usado para levitação, apenas supercondutores do tipo II são de uso prático. A combinação de forças devido ao aprisionamento de fluxo e forças repulsivas devido ao campo expelido faz com que ímãs e supercondutores exibam este comportamento.

Forças de levitação entre imãs e supercondutores de alta temperatura crítica podem apresentar

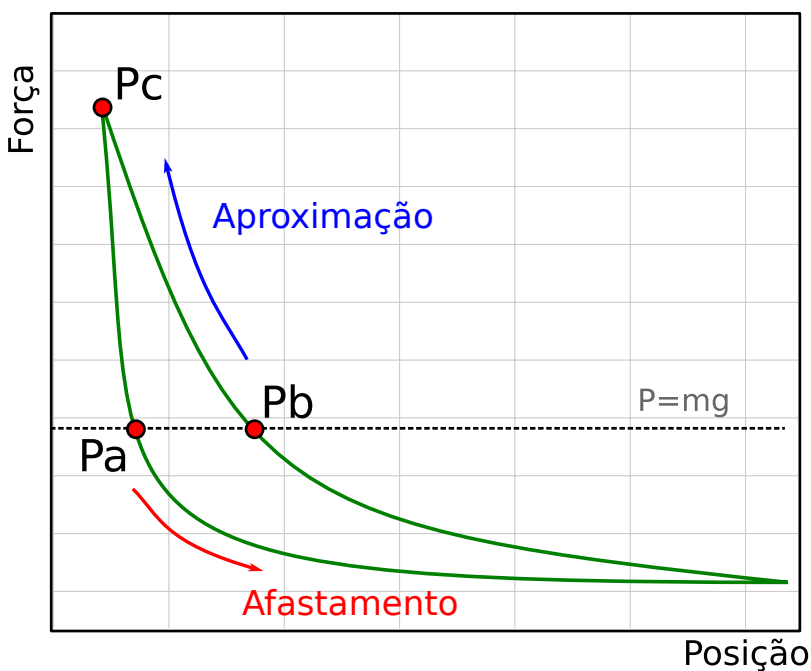

Figura 3: Forças em um ímã levitado por um supercondutor. 
caráter histerético e em alguns casos é possível notar uma força atrativa entre íma e o HTS.

O comportamento mostrado na figura 3 apresenta a forma de uma banana, dai o nome "força banana" para caracterizar este comportamento.

\section{O Projeto MagLev Cobra}

\subsection{Breve Histórico}

O desenvolvimento de um produto com base tecnológica, desde sua concepção até a disponibilidade final para uso seguro pela sociedade, passa por várias etapas de amadurecimento. As etapas seguidas pelo desenvolvimento do MagLev Cobra podem ser agrupadas em 4 grandes categorias, assim organizadas:

1. Prova de Conceito, que correspondeu à construção de um Protótipo em Escala Reduzida, de 2000 a 2006;

2. Protótipo Funcional, que correspondeu à construção de um primeiro protótipo com o objetivo de demonstrar a viabilidade técnica do veículo em escala real, sua capacidade de suportar cargas, efetuar curvas, vencer declividades. Tudo ainda dentro de um ambiente controlado de laboratório, de 2008 a 2012;

3. Protótipo Operacional, atual etapa do projeto, que corresponde à construção de um protótipo que opera semanalmente, em uma linha aberta com 200 metros de extensão, para qualquer usuário, dentro do campus da UFRJ. Em outras palavras, trata-se da comprovação da viabilidade operacional do equipamento (de 2012 a 2016).

4. Industrialização, a partir de 2016, cujos primeiros passos consistirão na certificação da tecnologia e na implantação de uma linha de $5 \mathrm{~km}$ de efetivo transporte urbano no campus universitário, com a necessidade da criação de uma cadeia produtiva para os diversos componentes do novo sistema de transportes.

A figura 4 apresenta a evolução do projeto MagLev Cobra ao longo dos últimos anos.

O desenvolvimento do veículo MagLev Cobra pode ainda, futuramente, incentivar diversas áreas de tecnologia, como por exemplo:

- Fabricação de imãs de terras raras com grande impacto na indústria eletro/mecânica;

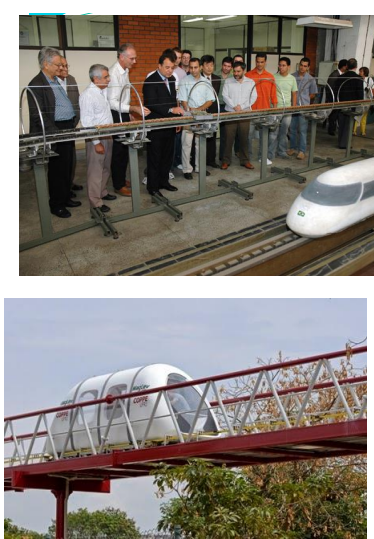

(C)

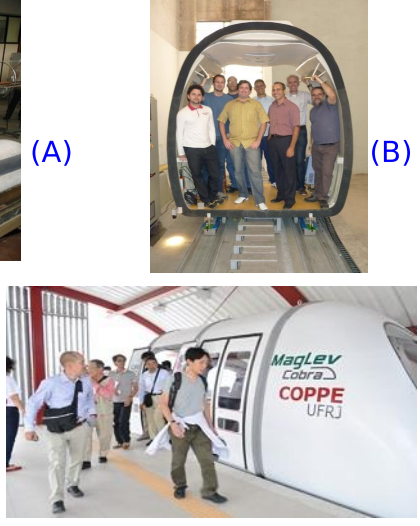

(D)
Figura 4: (A) Protótipo em escala reduzida, em 2000. (B) Protótipo em escala real, em 2011. (C) Protótipo em escala real operando numa linha aberta. (D) Protótipo usado para transporte no campus universitário em 2015.

- Processamento de materiais supercondutores para aplicações no setor elétrico (transformadores, geradores, limitadores de corrente, cabos, etc.);

- Fabricação de conversores de potência;

- Desenvolvimento de Mancais Magnéticos para ultra centrífugas, volantes de inércia, compressores e turbinas;

- Produção de Motores Lineares com variada aplicação industrial;

- Estabelecimento de novos padrões arquitetônicos e de transporte em grandes centros urbanos;

- Atração de jovens talentos para a carente área de ciência e tecnologia.

\subsection{Principais Vantagens do veículo MagLev}

As vantagens deste projeto são de três principais naturezas: construção civil e arquitetura, racionalização energética com menores emissões gasosas e operacional.

\subsubsection{Construção Civil e Arquitetura}

Veículos rodoviários e ferroviários utilizados no transporte público têm seu peso total transmitido ao solo através dos eixos, resultando em cargas concentradas.

No MagLev, o peso total é distribuído ao longo dos blocos supercondutores, resultando em cargas distribuídas. Esta configuração tem grande efeito no dimensionamento das colunas de sustentação 
para veículos que circulem sobre vias elevadas: o momento devido à flexão para uma carga concentrada no centro de uma coluna de sustentação é o dobro do valor com a mesma carga distribuída. O VLT, por exemplo,é um veículo que está sujeito às normas ferroviárias e, portanto, a estrutura da via não é muito diferente dos sistemas ferroviários convencionais. Considerando uma linha assentada sobre lastro de brita, dormentes de concreto e trilhos, o peso deste conjunto por metro linear é superior a $1.500 \mathrm{~kg}$.

Comparativamente, a estrutura de um veículo MagLev, ilustrada na figura 5, compõe-se de duas linhas de ímãs com $50 \mathrm{~mm}$ de altura e $100 \mathrm{~mm}$ de largura, correspondendo a um peso por metro linear inferior a $10 \%$ do peso da superestrutura do VLT. Além disso, uma estrutura mais leve custa muito menos.

Em relação ao veículo metroviário tradicional, o MagLev, que se inscreve em um círculo, possui características que podem gerar grandes reduções de custos, principalmente nos túneis em curva. Nestes trechos, onde se exige superelevação da linha, a escavação do túnel dos sistemas convencionais necessita acompanhar esta exigência, constituindo uma obra mais sofisticada do que um simples túnel circular.

Para veículos que necessitam do atrito da roda sobre trilho para trafegarem, a inclinação máxima permitida das vias é de 4\%. Decorrente da utilização de motores lineares para a tração do MagLev, esta limitação técnica é superada facilmente, sendo o limite máximo fixado pelo conforto dos passageiros (15\%). Então, a necessidade de construção de túneis e viadutos, bem como de estações subterrâneas, fica reduzido em regiões de topografia acidentada.

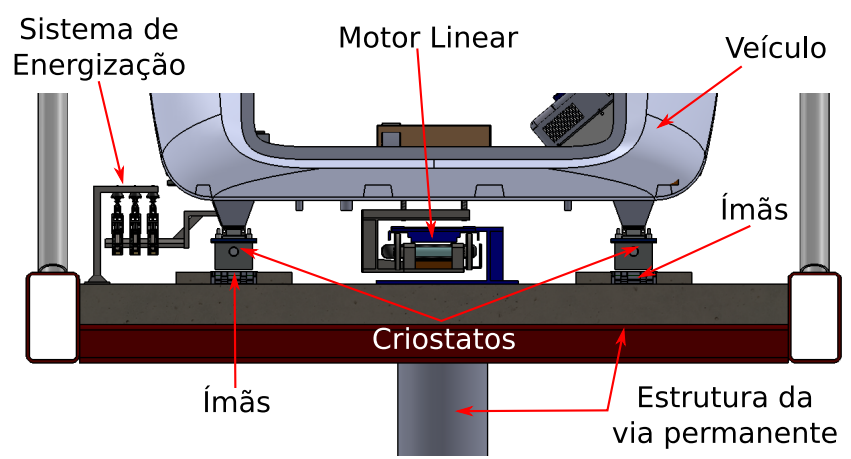

Figura 5: Principais componentes da estrutura da via permanente.
Os sistemas metroviários tradicionais exigem raios de curva superiores a $250 \mathrm{~m}$. Como o MagLev projetado tem múltipla articulação, este grande limite técnico para projetos de engenharia é também rompido, propiciando aos arquitetos maior liberdade para projetos inovadores. Nas aplicações urbanas, o MagLev pode, portanto, acompanhar perfeitamente as vias existentes, inserindo-se de maneira integrada no ambiente.

\subsubsection{Racionalização Energética com Menores Emissões Gasosas}

O MagLev Cobra não possui atrito entre rodas e trilhos, o que representa uma vantagem em relação ao transporte urbano convencional. Cálculos mostraram que o consumo energético na operação da linha de teste do veículo MagLev Cobra durante 4 horas diárias é tão baixo que pode ser suprido com três painéis fotovoltaicos (figura 6) 7 .

No que se refere a emissão de gases, os benefícios para o meio ambiente, decorrentes da substituição de parte do transporte individual (carros) pelo MagLev, serão imediatos, pois este opera com energia elétrica que, no Brasil, majoritariamente é gerada por fontes renováveis.

\subsubsection{Operacional}

Quando comparado a trens convencionais, o MagLev apresenta as seguintes vantagens de operação:

1. Redução do consumo de energia: A energia para levitar o veículo MagLev Cobra é apenas a necessária para refrigerar os supercondutores com nitrogênio líquido. Estes supercondutores são alojados dentro de criosta-

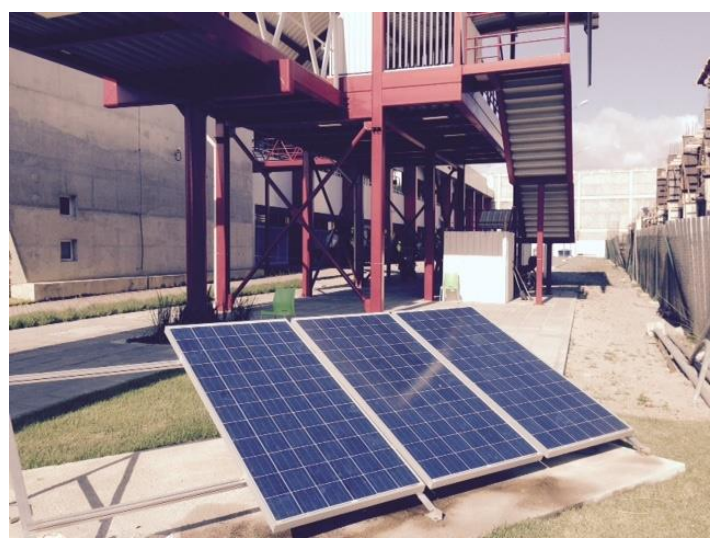

Figura 6: Alimentação com energia solar. 
tos e a recarga se faz apenas a cada 12 horas. Portanto, o consumo energético para levitar é baixo, quando comparado com os métodos de levitação eletrodinâmica ou eletromagnética. Para a tração, como a resistência é limitada ao atrito do ar e a velocidade é baixa $(¡ 70 \mathrm{~km} / \mathrm{h})$, o consumo médio de energia por passageiro é inferior, em comparação com outros meios de transporte.

2. Redução de ruído audível: Sem atrito e funcionando em velocidades baixas, o ruído produzido é insignificante e o veículo não perturba a vida da cidade, sendo apropriado para ser implantado em esbeltas linhas elevadas ao longo de rios, canais, canteiros centrais ou linha costeira.

3. Facilidade de mudança de via: a mudança de direção pode ser conseguida com eletroímãs, substituindo a lenta solução mecânica tradicional.

4. Menor tempo de viagem: com tração dada por motor elétrico linear, as forças para acelerar e frear não sofrem as limitações impostas pela aderência roda-trilho, permitindo, com isso, redução nos tempos de viagem.

5. Manutenção mais fácil: a ausência de contato mecânico torna a manutenção mais fácil do que a dos motores cilíndricos com rolamentos e desgaste. O trilho magnético também requer menos manutenção do que o sistema ferroviário tradicional com os procedimentos de alinhamento de dormentes e trilhos.

\subsection{Criostatos com Blocos de YBCO}

Os criostatos designados para operar como "rodas" do veículo MagLev Cobra devem apresentar as seguintes propriedades:

1. Minimizar a transferência de calor entre as partes internas e externas;

2. Suportar consideráveis forças e perturbações externas sem o surgimento de sérios danos;

3. Conter materiais supercondutores com grande campo crítico e boa qualidade mecânica;

4. Manter a distância entre os supercondutores no interior o mais próximo possível do campo magnético externo.

A minimização da transferência de calor tem um papel importante no aumento da eficiência energética do criostato. Existem diversos meios possíveis de refrigerar o material supercondutor dentro do criostato: utilizando um sistema de cryocooler de ciclo fechado com uma cabeça fria em cada dispositivo (criostato), ou utilizando uma central de resfriamento, ou ainda por transferência de calor diretamente com um fluido refrigerante (como o nitrogênio líquido) e os supercondutores (blocos de YBCO).

Para o caso do primeiro protótipo em tamanho real a utilização do líquido refrigerante na forma manual e com a saída livre para evaporação (vent) foi escolhida por ser mais simples de fabricar, mesmo sendo mais complicada de se utilizar em uma operação regular do veículo. No caso de um veículo utilizado para operação comercial, o sistema de ciclo fechado provavelmente, será a solução a ser implementada.

Os atuais criostatos empregados no protótipo MagLev Cobra foram projetados e construídos pela empresa alemã ATZ GMBD [8,9], visando-se satisfazer todos os quatro itens descritos acima. As figuras 7. A e 7B mostram fotografias e um desenho esquemático do corte transversal dos criostatos. Pode-se notar pela figura $7 \mathrm{~B}$ os detalhes do contêiner de $\mathrm{LN}_{2}$ e blocos supercondutores.

A distribuição dos blocos de YBCO dentro de cada criostato está ilustrada na figura $8 \mathrm{~A}$. Ao todo, cada criostato possui 24 blocos de YBCO que são

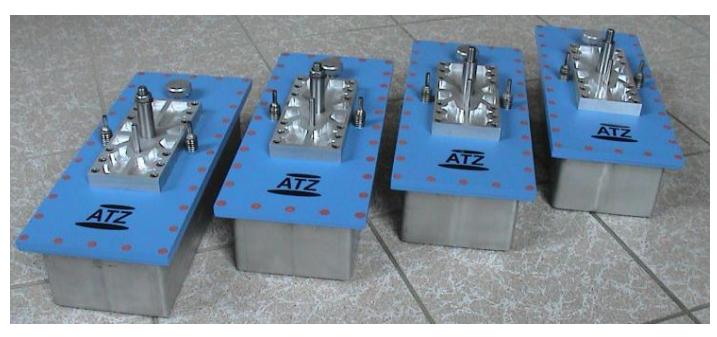

(A)

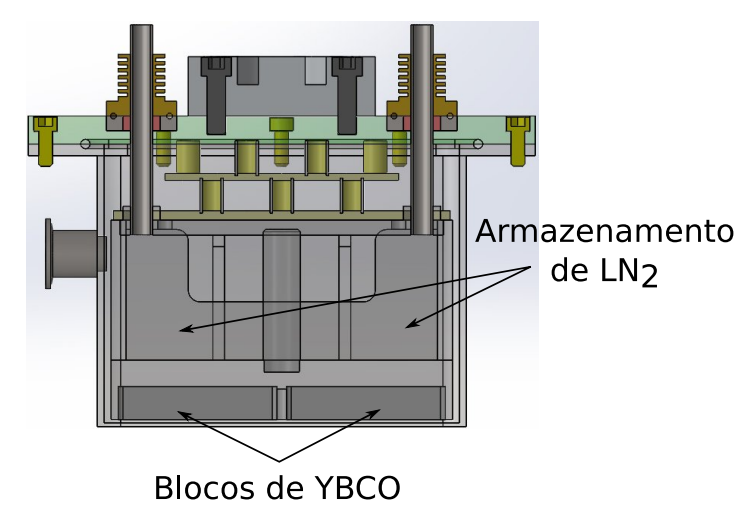

Figura 7: (A) Alguns criostatos usados no projeto e (B) corte transversal de um criostato, enaltecendo a reserva de nitrogênio líquido e os blocos supercondutores 
divididos em duas colunas de 12 blocos, como mostra a figura $8 \mathrm{~A}$.

Os blocos de YBCO consistem em materiais texturizados, com três sementes de nucleação que permitem a criação de 3 pequenos laços de corrente dentro de cada domínio supercondutor e um laço adicional envolvendo os três domínios (figura 8C) [10].

\subsection{Trilhos de Ímãs}

Uma vez que os criostatos são pré-definidos pelo fabricante, foi necessário o desenvolvimento de um trilho de ímãs permanente que, por um lado maximizasse a força de levitação e por outro lado minimizasse a quantidade de ímãs permanentes usados na linha.

Criostatos e trilhos, quado operado juntos, formam um grupo que na literatura é conhecido como mancais magnéticos supercondutores (SMB - Superconducting Magnectic Bearings). A fim de avaliar a força de levitação e as propriedades do SMB, diversos protótipos de trilho foram testados ao longo do desenvolvimeto do projeto. $\mathrm{O}$ primeiro trilho protótipo construído era feito com ímas de ferrite 11.12. O custo destes ímãs foi a principal razão para esta primeira tentativa. A força de levitção do SMB com estes imãs porém não era suficiente para um trem de levitação.

O baixo custo e produção em larga escala de ímãs de Neodímio-Ferro-Boro (Nd-Fe-B) na China entre 2003 e 2008 motivou a investigação destes ímãs no projeto MagLev Cobra. Além disso, estes ímãs apresentam melhores parâmetros para aplicações que visam levitação, tais como: densidade de fluxo magnético, força coercitiva e coercitividade intrínseca 13. O primeiro protótipo do MagLev Cobra usando ímas Nd-Fe-B foi construído em pequena escala numa linha de $30 \mathrm{~m}$ de extensão [14].
A construção deste protótipo em pequena escala foi fundamental para que se pudesse determinar a melhor configuração dos trilhos de ímãs. Foi a partir deste protótipo que modelos de simulação foram desenvolvidos para cálculos de força de levitação e estabilidade lateral [13, 15 18].

As simulações foram essenciais para a determinação da melhor geometria de disposição dos ímãs ao longo do trilho. A otimização do trilho considerou o preço dos ímãs, uma força de levitação de $2500 \mathrm{~N}$ por criostato e um gap de levitação entre $8 \mathrm{~mm}$ e 10 $\mathrm{mm}$ [19. Finalmente, a geometria de disposição dos ímãs para a formação do trilho, é aquela ilustrada na figura 94. As setas indicam a direção de magnetização. O ferro entre os ímãs servem para uma melhor concentração de fluxo.

A figura $9 \mathrm{~B}$ mostra uma fotografia da construção dos trilhos de acordo com a geometria escolhida.

\subsection{Sistema de Tração}

Veículos de levitação são tracionados através de motores lineares (ML). Isso se deve às imposições construtivas deste tipo de tecnologia, que necessita de tração direta sem aparatos de translação de movimento.

Os trens de levitação projetados para operarem dentro de cidades, caracterizados por serem de baixa velocidade (em torno de $70 \mathrm{~km} / \mathrm{h}$ ), utilizam motores de indução linear (MIL), entretanto, veículos de alta velocidade (que podem superar $500 \mathrm{~km} / \mathrm{h}$ ), utilizam motores síncronos lineares (MSL). A escolha do tipo de motor se deve, entre outras características, aos custos associados a cada topologia e suas aplicações.

O MagLev Cobra utiliza para sua tração um MIL de primário curto e secundário longo, conforme ilustrado na figura 10. Primário é a parte do motor que contém os enrolamentos de fase e estão embarcados (instalados) no veículo. O secundário se estende ao

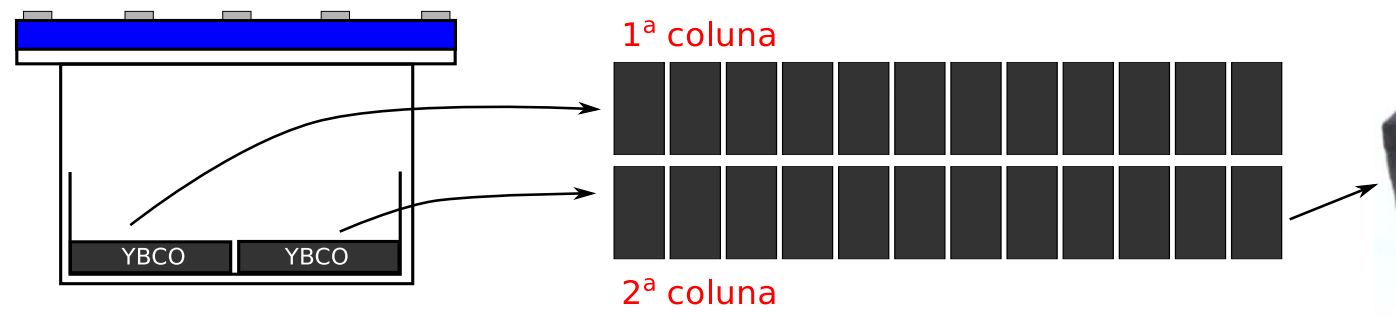

(A)

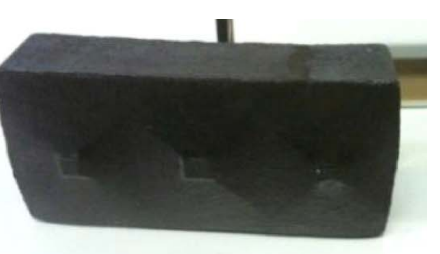

(C)

Figura 8: (A) Posição dos blocos de YBCO dentro do criostato. (B) Disposição em duas colunas de 12 blocos (total de 24 blocos) e (C) Fotografia de um bloco texturizado de YBCO com três sementes de nucleação. 

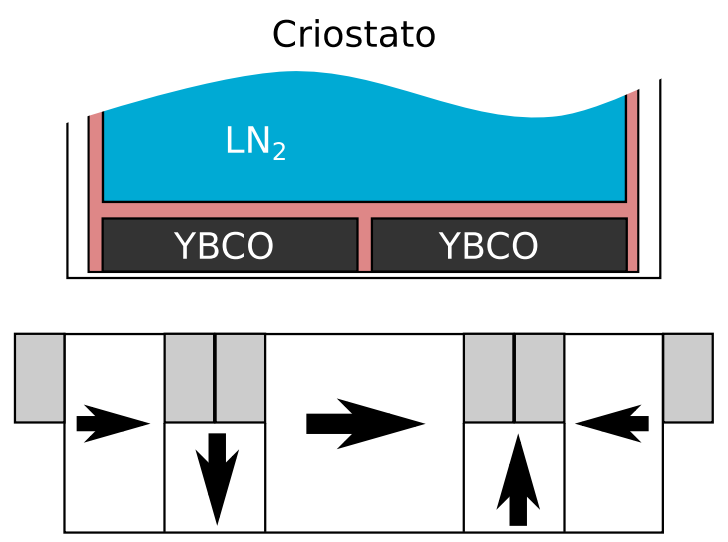

$\square$ Ferro $\quad \square$ Ímã

(A)

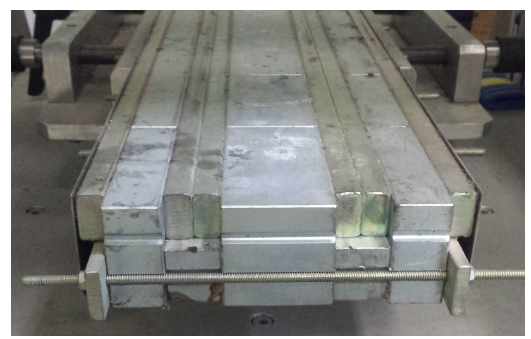

(B)

Figura 9: (A) Ilustração da disposição de ímãs ao longo do trilho e (B) fotografia da construção dos trihos.

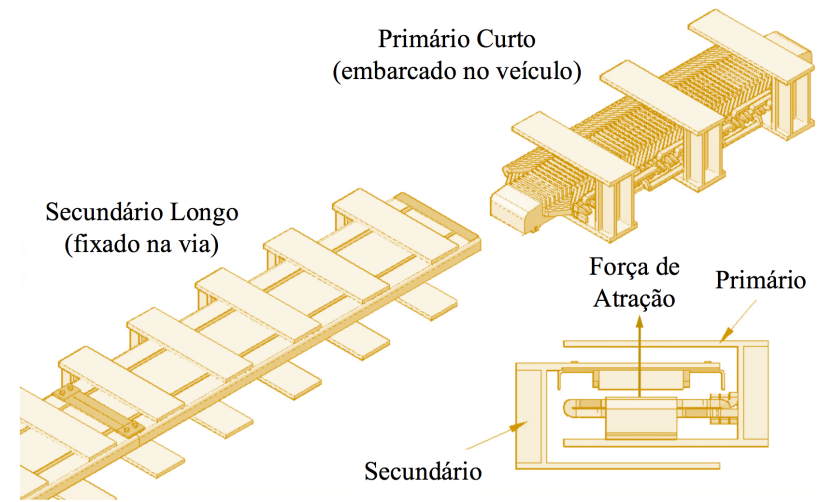

Figura 10: Motor de Indução Linear.

longo do trajeto e é composto por lâminas de ferro e barras de alumínio curto-circuitadas.

A topologia do MIL, primário curto ou longo, pode variar a cada projeto e a quantidade de primários em cada trem também obedecerá as características de cada aplicação. Dimensões do motor e os dados elétricos estão apresentados na tabela 1 .

Modelos computacionais, bidimensionais e tridimensionais, permitem calcular as forças desenvolvi-
Tabela 1: Parâmetros do MIL do MagLev-Cobra.

\begin{tabular}{lc}
\hline Parâmetros & MagLev-Cobra \\
\hline Tensão & $420 \mathrm{~V}-(\mathrm{Y})$ \\
Corrente & $53 \mathrm{~A}$ \\
Frequência & $25 \mathrm{~Hz}$ \\
Potência & $10 \mathrm{HP}$ \\
Número de Pólos & 6 \\
Passo Polar, $\tau$ & $156.0 \mathrm{~mm}$ \\
Entreferro & $8 \mathrm{~mm}$ \\
Densidade ${ }^{1} F_{x}$ & $52.5 \times 10^{3} \mathrm{~N} / \mathrm{m}^{3}$ \\
\hline Primário & MagLev-Cobra \\
\hline Comprimento & $1270 \mathrm{~mm}$ \\
Altura & $106 \mathrm{~mm}$ \\
Largura do Núcleo & $166 \mathrm{~mm}$ \\
Largura do Enrolamento & $340 \mathrm{~mm}$ \\
Índice de Proteção & $I P-23$ \\
Peso & $132 \mathrm{~kg}$ \\
\hline Secundário & MagLev-Cobra \\
\hline Comprimento & $1510 \mathrm{~mm}$ \\
Altura & $53 \mathrm{~mm}$ \\
Largura núcleo e enrolamento.) & $231 \mathrm{~mm}$ \\
Peso & $53.98 \mathrm{~kg} / \mathrm{m}$ \\
\hline Densidade de Força (volume do primário: $\mathrm{c} \times \mathrm{a} \times l$
\end{tabular}

das pelo motor através de métodos por elementos finitos 20], conforme mostra a figura 11

Os resultados obtidos através de simulações computacionais e experimentos em laboratórios estão apresentados na tabela 2. A força de atração entre o primário e o secundário do motor contribui com a levitação do veículo, conforme a figura 10 evidencia. A atração entre as partes do MIL age como uma parcela somatória à força desenvolvida pelos criostatos 21.

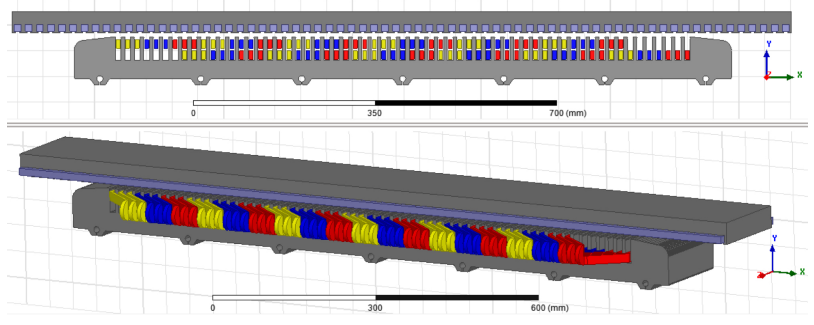

Figura 11: Modelo em 2D e 3D do MIL utilizado no MagLev Cobra.

Tabela 2: Resultados Compilados

\begin{tabular}{lccc}
\hline Força $^{1}$ & 2D & 3D & Experimental \\
\hline Tração (N) & 2489.70 & 1908.00 & 2403.00 \\
Atração (N) & 2987.00 & 4197.00 & 2998.00 \\
Lateral (N) & - & 436.00 & - \\
\hline \multicolumn{4}{c}{${ }^{1}$ entreferro $=8 \mathrm{~mm}$}
\end{tabular}




\subsection{Testes Operacionais}

A atual linha de testes foi aberta ao público a partir da Semana Nacional de Ciência e Tecnologia (outubro de 2015). A partir de então, visitas regulares são conduzidas uma vez por semana, no intervado de uma hora, transportando alunos e professores da universidade entre os prédios do Centro de Tecnologia (CT1 e CT2). Desde o início de sua operação até a presente data (Junho de 2016) mais de 2000 pessoas já foram transportadas pelo protótipo do MagLev Cobra.

Cada viagem dura 2 minutos e são transportados, no máximo, 10 passageiros. A velocidade máxima, no trecho de 200 metros é de $10 \mathrm{~km} / \mathrm{h}$.

Como passos futuros, objetiva-se desenvolver o projeto de viabilidade técnica, econômica e ambiental da construção da linha de $5 \mathrm{~km}$, ao longo do campus da universidade. Nesta linha, a velocidade poderá atingir $100 \mathrm{~km} / \mathrm{h}$ entre as estações mais distantes, ou em viagens expressas (sem paradas).

\section{Conclusões}

Este artigo mostrou que a tecnologia supercondutora de levitação utilizada no MagLev Cobra para transporte urbano é correta em todos os aspectos, a saber:

- Ecologicamente, por ser um veículo não poluente, devido ao baixo barulho produzido e também devido ao baixo consumo de energia;

- Economicamente, devido aos baixos custos de manutenção e implantação;

- Tecnicamente, pois o método de levitação empregado é adequado para transportes de baixa velocidade;

- Socialmente, uma vez que oferece solução para o problema de transporte em grandes cidades.

Além disso, qualidade de vida juntamente com crescimento industrial, técnico e científico são favorecidos com este projeto.

\section{Agradecimentos}

Ao suporte financeiro da FAPERJ, BNDES, CAPES, CNPq e Secretaria de Assuntos Estratégicos (SAE).

À contribuição para o desenvolvimento deste trabalho recebida dos seguintes pesquisadores e técnicos: Antonio C. Ferreira, Guilherme G. Sotelo, Daniel H.
N. Dias, Felipe Sass, Eduardo S. Motta, Edeval V. Gangá, Renato P. S. Filho, Jonathan G. Tavares e Severina Guedes.

\section{Referências}

[1] R.M. Stephan, R. de Andrade Jr. and A.C. Ferreira, IEEE Veh. Technol. Mag. 7, 122 (2012).

[2] J.G. Bednorz and K.A. Müller, Z. Physik, B 64, 18 (1986).

[3] Transrapid, acessado em 11/6/2016, disponível em http://www.maglev.com

[4] MagLev Systems, acessado em 11/6/2016, disponível em http://www.rtri.or.jp.

[5] Shangai Maglev Transportation, acessado em 11/6/2016, disponível em http: //www.smtdc.com/ en/index.html

[6] T.P. Sheahen, Introduction to High-Temperature Superconductivity (Plenum Press, New York, 1994).

[7] V.R. de Moura, Análise Energética do Módulo de Transporte do MagLev Cobra. Monografia Engenharia Elétrica, Universidade Federal do Rio de Janeiro, 2006.

[8] F.N. Werfel, U. Floegel-Delor, T. Riedel, R. Rothfeld, D. Wippich and B. Goebel, IEEE Trans. Appl. Supercond. 20, 874 (2010).

[9] F.N. Werfel, U. Floegel-Delor, T. Riedel, R. Rothfeld, D. Wippich, B. Goebel, and P. Schirrmeister, Supercond. Sci. Technol. 25014007 (2012).

[10] Z. Deng, M. Izumi, M. Miki, K. Tsuzuki, B. Felder, W. Liu, J. Zheng, S. Wang, J. Wang, U. FloegelDelor and F.N. Werfel, IEEE Trans. Appl. Supercond. 22, 6800210 (2012).

[11] R. Stephan, R. de Andrade Jr., G. dos Santos, M. Neves and R. Nicolsky, Phys. C Supercond. 386,490 (2003).

[12] R. de Andrade Jr., J.A. A.S. Cardoso, G.C. dos Santos, L.B. de Cicco, A.F.G. Fernandes, M.A.P. do Rosário, M.A. Neves, A.P. de Souza, A. Ripper, G.C. da Costa, R. Nicolsky, and R.M. Stephan, IEEE Trans. Appl. Supercond. 13, 2271 (2003).

[13] E.S. Motta, Otimização de Trilho Magnético de um Sistema de Levitação Supercondutora para Veículo Maglev. Tese de Doutorado, COPPE/UFRJ, 2011.

[14] R. Stephan, R. Nicolsky, M. Neves, A. Ferreira, R. de Andrade Jr., M.C. Moreira, M. Rosario and O. Machado, Phys. C Supercond. 408, 932 (2004).

[15] G.G. Sotelo, Modelagem de Supercondutores Aplicada ao Projeto de Mancais Magnéticos. Tese de Doutorado, COPPE/UFRJ, 2007.

[16] G.G. Sotelo, R. de Andrade Jr. and A.C. Ferreira, IEEE Trans. Appl. Supercond. 19, 2083 (2009).

[17] D.H.N. Dias, Modelagem de Mancais Lineares Supercondutores Considerando o Resfriamento na Presença de Campo Magnético. Tese de Doutorado, COPPE/UFRJ, 2009. 
[18] D.H.N. Dias, E.S. Motta, G.G. Sotelo, J. Rubens de Andrade, R.M. Stephan, L. Kuehn, O. de Haas and L. Schultz, IEEE Trans. Appl. Supercond. 19, 2120 (2009).

[19] E.S. Motta, D.H.N. Dias, G.G. Sotelo, H.O.C. Ramos, J.H. Norman and R.M. Stephan, IEEE Trans. Appl. Supercond. 21, 3548 (2011).

[20] R.A.H. de Oliveira, T. Espenhahn, D. Berger, L. Schultz, A.C. Ferreira and R.M. Stephan, in: Proceedings of the 10th International Symposium on Linear Drives for Industry Applications (LDIA 2015), Aachen, 2015.

[21] R.A.H. de Oliveira, Sistema de Frenagem Regenerativa com Motor de Indução Linear do Veículo Maglev-Cobra. Dissertação de Mestrado, COPPE/UFRJ, 2013. 